\title{
A Method for Local Tuning of Fuzzy Membership Functions
}

\author{
Ahmet Çinar \\ Firat University, Faculty of Eng. Computer Engineering , 23119, Elazig, Turkey \\ acinar@firat.edu.tr
}

\begin{abstract}
In this paper, a new method based on genetic algorithms is proposed for local tuning of fuzzy membership functions. For this purpose, the local adjustment is employed on the initial membership functions. Genetic algorithm is used to investigate discrete points that will be modified on the membership functions Hence, global adjustment does not require and the processing time required for tuning of membership functions is decreased.
\end{abstract}

\section{Introduction}

In this paper, the initial base values of membership functions are not changed, only local modifications are made. The basic of the study is as following:

Step 1: Discretization of initial membership functions. Step 2: Finding the membership functions that will be modified by genetic algorithms and tuning. Step 3: Reconstructing the membership functions and applying to system.

\section{Discretization of Initial Membership Functions}

For this process, the geometric support construction method is used. The set of polygonal segments of boundary curve is located in the any list. And then, the construction of the polygonal support is achieved such that the distance any segment and portion of the curve it represent must be bounded. Hence, refinement of curve will be more important and correct in the any highly curved regions on the boundary curve.

\section{Tuning Membership Functions by Using Genetic Algorithms}

Figure 1 depicts diagram of proposed method for local tuning of fuzzy membership functions.

The following sections describe each block seen above.

Block 1: Dedicating control points to membership functions.

Block 2: Fitness Function.

In this block, how to make changes on the population is described. For this goal, initial membership function is passed through certain fitness function and the obtained 


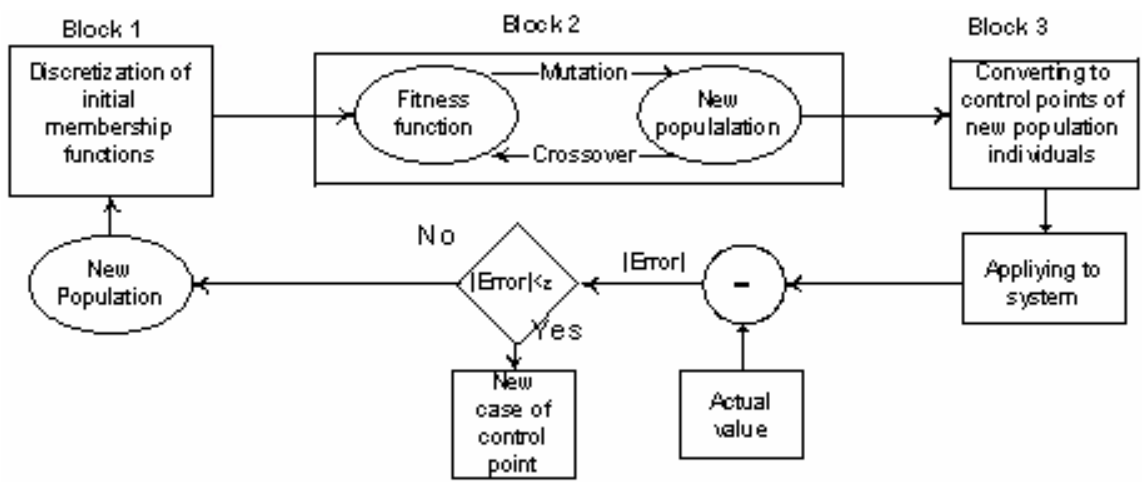

Fig. 1. The diagram of the proposed method

value is operated to genetic operator. Therefore, some individuals are founded. In this study, a function dependent on system input output is used as fitness function. It is known that, a fitness function is generated a certain integer value. Whereas, this method is not appropriate for this study due the fact that the direct fitness function did not explained. The following section describes the proposed fitness function. Figure 5 depicts genetic individuals and control points. These control points are produced by means of aforementioned method in block 1 . This process is the population dedicating process on the control points and making up control points on the initial membership function.

For example; let control point value be 11. First, log value of control point is obtained, such as $m=\log 2(11)=3.3$. If $m$ is not integer number, it is rounded for example 3.3 to 4 . Therefore, population values are follows: $0001,0010,0011,0100,0101,0110,0111,1001,1010,1011,1100$. The $m$ value is used as bit number.
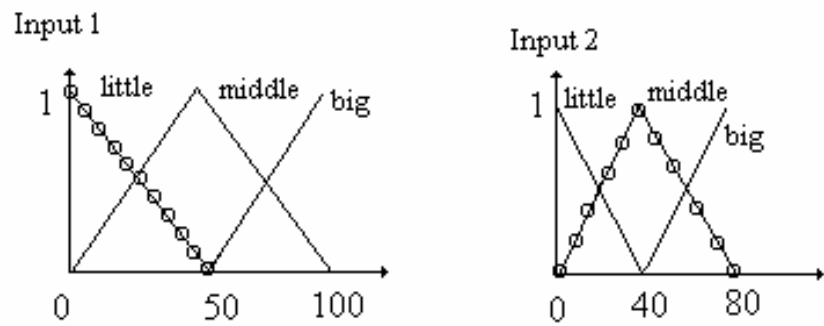

Fig. 2. Control points on the initial membership function

If the rule base table and system input output response are known, fitness function is used as following: Let us know the input output response for any system. $a[], \mathrm{b}[\mathrm{]}$ and $c[]$ are three sets. Let $c[]$ be response with respect to $a[]$ and $b[]$. Control points at distance $\varepsilon_{l}$ is changeable. But, we have to know which points be modified. 
For that reason, $a[]$ and $b[]$ sets are used. Figure 3 shows control points that will change with respect to $\varepsilon_{l}$ value.
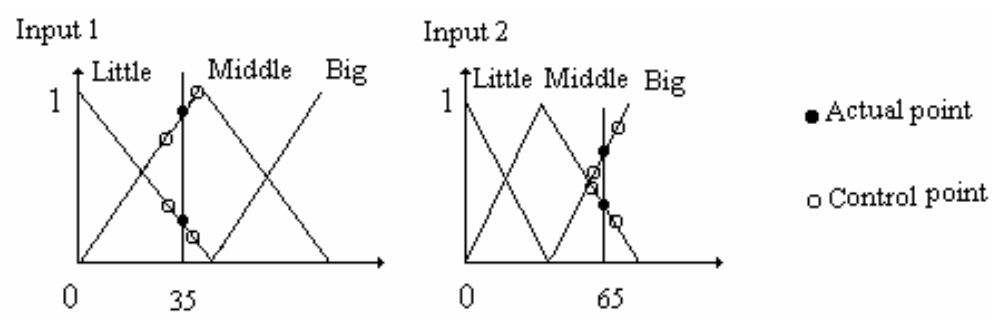

Fig. 3. The values with respect to input $=35$ and input $2=65$ and relationship control points

After finding these control points, the output value is obtained by means of any defuzzyfication method such as middle of maximum.

$u_{i}$ : The $i_{\text {th }}$ value of $u$ membership functions

$\mu\left(u_{i}\right)$ : The value of $u_{i}$ membership functions.

$$
u^{*}=\frac{\sum_{i=1}^{l} \cdot \mu\left(u_{i}\right) \cdot u_{i}}{\sum_{i=1}^{l} \mu\left(u_{i}\right)}
$$

If the obtained result is similar to output $c\left[\right.$ ] with respect to $\varepsilon_{l}$, then any changes does not require on this population. In the proposed method, value $\varepsilon_{l}$ is as half of distance between of two points as shown in figures 4 . If the error is bigger than $\varepsilon_{l}$, then generic operators are used on population. Figure 4 depicts simple geometric operations for value $\varepsilon_{1}$.
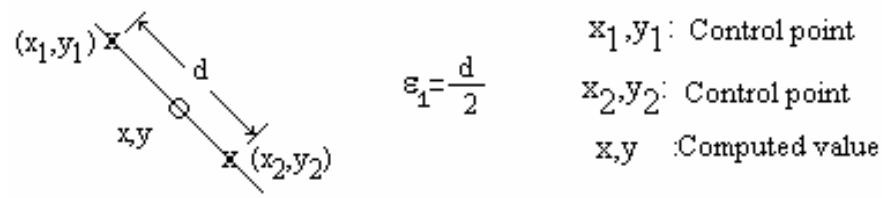

Fig. 4. The using method for value $\varepsilon l$

The mutation is applied to discrete points changed. It is known that, only a bit is changed in the simplest manner for the mutation process. A new population after this operation will be different. This new population has to convert in the appropriate for system. This operation is achieved in block 3 . 
Block 3: Converting to control points of new population value.

In this block, new population values are converted to control points. Let original point be genetic individual with respect to 0010 value. Figure 5 shows this position.

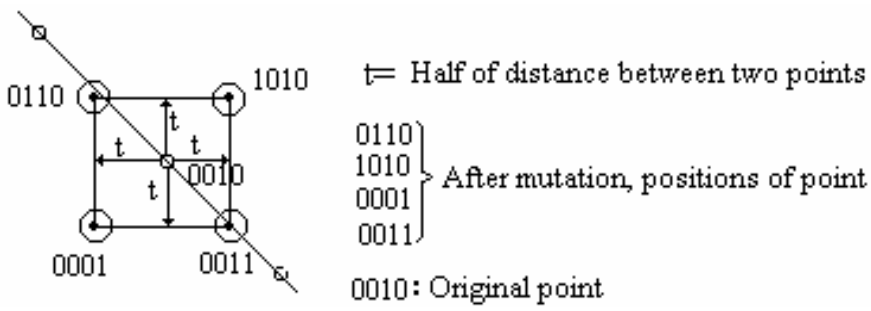

Fig. 5. Original point and its position after modification

According to using bit numbers, the actual location of point is as follows:

$\mathrm{x}, \mathrm{y}$ value is $0010, \mathrm{x}+\mathrm{t}, \mathrm{y}+\mathrm{t}$ value is $0011, \mathrm{x}-\mathrm{t}, \mathrm{y}+\mathrm{t}$ value is $0001, \mathrm{x}-\mathrm{t}, \mathrm{y}-\mathrm{t}$ value is $0110, x+t, y-t$ value is 1010 . If bit number is 4 , there is no problem. But the bit number is bigger than 4 , then only right 4 bits are used. Also, let bit number be 5 and 00100 . Then only using 0100 is appropriate. If bit number is less than 4 , then, a bit is added on the left side. If bit value is 010 , then it is used as 0010 .

In this way, after computing actual coordinate values and making up memberships functions, according to system response, modifications of membership function is considered. If it needs, new population is passed through fitness function.
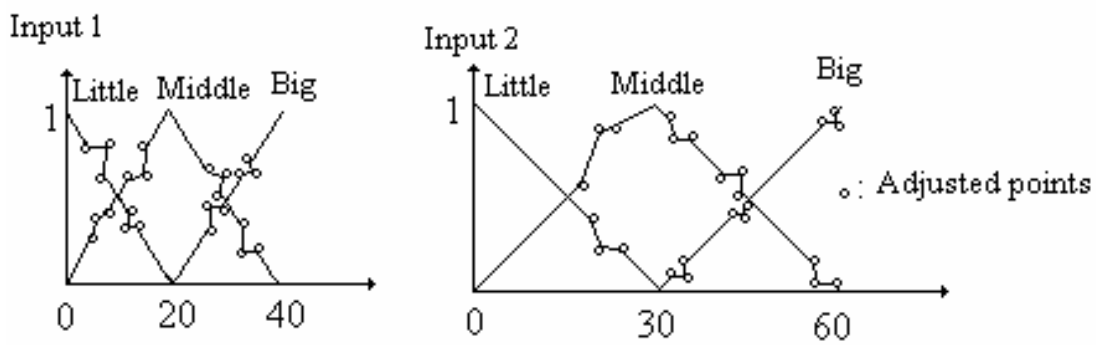

Fig. 6. The adjusted membership functions

\section{Conclusion}

In this study, a distinct method based on genetic algorithm for local adjustment of fuzzy membership functions is presented. Instead of global adjustment, local adjustment is achieved. Thus, the fast and accurate process is achieved with respect to system input-output response. By means of proposed method, initial fuzzy membership functions are selected, and then very much little changes on the 
membership function are done. Consequently the global changes such as gradientdescent method are avoided.

\section{References}

1. D.E. Goldberg, Genetic Algorithms in Search, Optimization and Machine Learning, Addison Wesley, 1989

2. H. Nomura, I. Hayashi, N.Wakai, A self-tuning method of fuzzy control by descent method, in Proc $4^{\text {th }}$ IFSA congress, Engineering, pp.49-52, 1991

3. H.J.Zimmermann ,Fuzzy programming and linear programming with several objective functions, Fuzzy Sets and Systems, vol.1, pp.44-45,1978

4. Kuo Ting, Hwang Shu-Yuen, A Genetic Algorithm With Disruptive Selection, IEEE Transaction On Systems, Man, And Cybernetics-Part B: Cybernetics, Vol. 26, No. 2, April 1996

5. Wang Chi-Hsu,Wang Wei-Yen, Fuzzy B-Spline Membership Function (BMF) And Its Application In Fuzzy-Neural Control, IEEE Transactions On System Man And Cybernetics, Vol. 25, No.5, May 1995

6. Zadeh L., Fuzzy sets, Inf. Control, Vol:8, pp:338-353, 1965. 